\title{
BMJ Open Brief strategic therapy for obsessive- compulsive disorder: a clinical and research protocol of a one-group observational study
}

\author{
Giada Pietrabissa, ${ }^{1,2}$ Gian Mauro Manzoni, ${ }^{1,3}$ Padraic Gibson, 4,5,6,7,8 \\ Donald Boardman, ${ }^{8}$ Alessio Gori, ${ }^{9}$ Gianluca Castelnuovo ${ }^{1,2}$
}

To cite: Pietrabissa $\mathrm{G}$, Manzoni GM, Gibson P, et al. Brief strategic therapy for obsessive-compulsive disorder: a clinical and research protocol of a onegroup observational study. BMJ Open 2016;6:e009118. doi:10.1136/bmjopen-2015009118

- Prepublication history for this paper is available online. To view these files please visit the journal online (http://dx.doi.org/10.1136/ bmjopen-2015-009118).

Received 18 June 2015 Revised 2 December 2015 Accepted 16 December 2015

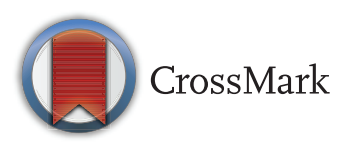

For numbered affiliations see end of article.

Correspondence to Dr Giada Pietrabissa; giada.pietrabissa@unicatt.it

\section{ABSTRACT}

Introduction: Obsessive-compulsive disorder (OCD) is a disabling psychopathology. The mainstay of treatment includes cognitive-behavioural therapy (CBT) and medication management. However, individual suffering, functional impairments as well as the direct and indirect costs associated with the disease remain substantial. New treatment programmes are necessary and the brief strategic therapy (BST) has recently shown encouraging results in clinical practice but no quantitative study has as yet been conducted.

Methods and analysis: The clinical effectiveness of the OCD-specific BST protocol will be evaluated in a one-group observational study. Participants will be sequentially recruited from a state community psychotherapy clinic in Dublin, Ireland. Outcome measures will be the Yale-Brown Obsessive Compulsive Scale (Y-BOCS) and the Beck Depression Inventory-II (BDI-II). Data will be collected at baseline, at treatment termination and at 3 month follow-up. The statistical significance of the post-treatment effect will be assessed by the paired-sample Student $t$ test, while clinical significance will be evaluated by means of the equivalence testing method, which will be also used to assess the maintenance of effect at follow-up.

Ethics/dissemination: The present study is approved by the Hesed House Ethics Board in Dublin. Findings will enhance the evidence-based knowledge about the clinical effectiveness of BST in treating OCD symptoms, prior to assessing its efficacy in a randomised and controlled clinical trial, and will be disseminated through publication in peer-reviewed journals and conference presentations.

\section{INTRODUCTION}

Obsessive-compulsive disorder (OCD) is an anxiety syndrome characterised by the presence of recurrent or persistent thoughts, impulses or images (obsessions) that are experienced as intrusive or distressing by the person, and that he or she attempts to ignore or suppress by performing repetitive

\section{Strengths and limitations of this study}

- The observational study design allows the assessment of treatment effects in real-world settings.

- A positive result in an observational study can inform practice directly.

- The lack of a concurrent control group limits the internal validity of results.

- A convenience sampling limits the generalisability of results.

behaviours or mental acts (compulsions). ${ }^{1}$ Symptoms usually begin gradually, tend to vary in severity throughout the individual's life, and generally worsen when intense stress is experienced by the person. OCD, considered a lifelong disorder, can be so severe and time-consuming to the point of significantly interfering with the person's normal routine, occupational (or academic) functioning, or usual social activities or relationships. ${ }^{2}$ Epidemiological studies report a lifetime disorder's prevalence of $1-4 \%$ in the general population, ${ }^{3-5}$ equal for men and women, although it is more commonly diagnosed among boys than girls. ${ }^{4}$ The Global Burden of Diseases study has recently ranked OCD as the 10th leading cause of disability worldwide $^{7}$ and it is also considered the fourth most common mental illness in many western countries.

Most adults recognise that their obsessions and compulsions do not make sense, but that is not always the case. In addition, children may not realise that something is wrong, and too often even healthcare professionals do not identify the need for appropriate interventions, ${ }^{8}$ contributing to make OCD a very difficult-to-treat disorder. Sufferers of OCD generally display many non-OCD symptoms, such as signs of 
depression, ${ }^{9}$ excessive worry, extreme tension ${ }^{10}$ as well as severe occupational, social and family dysfunction. ${ }^{4} 1112$ Aside from the compulsive behaviours, there are no physical symptoms of OCD; however, OCD sufferers can develop physical problems. For example, in the presence of germ obsession, they may wash their hands so much as to make their skin red, raw and painful.

Most people with OCD fall into the washers category. They are afraid of contamination and usually have cleaning or hand-washing compulsions. Checkers, instead, repeatedly check things (oven turned off, door locked, etc) that they associate with harm or danger, and hoarders fear that something bad will happen if they throw anything away. They compulsively hoard things that they do not need or use. Another OCD pattern is the pathological doubt that if everything is not perfect or done just right, something terrible will happen, or they will be punished (doubters and sinners). Counters and arrangers, in addition, are obsessed with order and symmetry and they may have superstitions about certain numbers, colours or arrangements.

Most people with OCD present with both obsessions and compulsions, but some persons may experience just one or the other. OCD symptoms manifestation also vary greatly from individual to individual, and access to flexible, innovative, affordable and evidence-based psychological treatments for OCD is required.

\section{Current treatments for OCD}

There are different ways to treat OCD. These include psychotherapies, drugs (antidepressants) or a combination of both.

Cognitive-behavioural therapy (CBT) combined with antidepressant medication currently represents the best treatment option for OCD. ${ }^{13}$ This blended intervention does not provide a 'cure' for OCD, but controls the symptoms and enables people with OCD to restore normal functioning in their lives.

CBT refers to two distinct treatments: exposure and response prevention (ERP) therapy and cognitive therapy (CT). ${ }^{14}$ Although these treatments are increasingly offered in combination, they will be discussed separately.

Before starting ERP treatment, patients make a 'hierarchy' of situations that provoke in them obsessional fears; then they participate in exposure tasks, and subsequently they are asked to pay particular attention to thoughts and feelings related to these situations. ${ }^{15}$

ERP treatment involves a direct or imagined, controlled exposure to objects or situations that cause the patient mild-to-moderate anxiety. Over time, exposure to obsessional cues helps the individual to gradually 'get used' to (or habituated) to them, leading to reduction in anxiety. Exposure tasks are generally first performed with the therapist assisting, and sessions usually take between $45 \mathrm{~min}$ and $3 \mathrm{~h}$. Patients are also asked to practise exposure tasks between sessions for 2-3 h/day.
The main goal during both in vivo and imagery exposure is essentially for the person to stay in contact with the obsessional trigger without engaging in ritual behaviours, ${ }^{16-18}$ and the treatment duration depends on the patient's ability to tolerate anxiety and to resist compulsive behaviours. ${ }^{19}$

CT is focused instead on how participants interpret their obsessions: what they believe or assume to be true about them, what their attitude is towards them and why they think they have these obsessions. It is essentially aimed at helping participants to identify and re-evaluate beliefs about the potential consequences of engaging or not engaging in compulsive behaviour, so as to address it.

CBT, particularly ERP, was found to be effective in a number of clinical trials. ${ }^{4}$ Findings have shown CBT as being able to address OCD symptoms more than pharmacotherapy and to guarantee good follow-up rates of success among respondents. ${ }^{4} 6$ Notably, despite showing lower relapse rates than pharmacotherapy $(12 \%$ vs 24-89\%), for its own nature CBT causes people anxiety, and about $25 \%$ of patients drop out before termination or refuse the treatment. ${ }^{4}{ }^{12}$ Specifically, patients presenting with more obsessions than compulsions may confront serious difficulties with CBT, since some techniques can maintain the obsessions by turning the attention of the individual to the preoccupations themselves. 620

Studies documenting the benefits of ERP have found that $75 \%$ of respondents experience enhancement in OCD symptoms during the treatment, the majority of them showing continuous improvements even after 3 years from the end of the intervention. However, only $25-40 \%$ of patients reach full recovery, while most of them remain long-term symptomatic. ${ }^{21}$ The main reasons for failure of CBT include patients' lack of motivation in reducing rituals and the presence of comorbid disorders, such as moderate-to-severe depression or avoidant personality disorder. ${ }^{22}$ Unfortunately, even with effective medication, responders who suffer from severe symptoms show residual impairments ${ }^{9}$ and there are also serious health concerns with long-term pharmacotherapy usage.

At present, no treatment has been demonstrated to be totally curative for OCD. Most interventions can be expected to reduce symptoms by $50-80 \%$. However, the illness is cyclic, and worsens when the individual is under stress. Additional treatment strategies are thus required to more effectively tailor this complex symptomatology.

\section{The advanced model of brief strategic therapy (BST)}

According to the Brief Strategic treatment for OCD, if the disorder is not completely 'solved', symptoms tend to reoccur, and empirical evidence shows BST as being particularly effective in guaranteeing long-term stability of therapeutic outcomes. ${ }^{23}$ 
Clinical evidence demonstrates that BST is effective in treating several forms of psychological suffering, including OCD. The Brief Strategic approach makes a selfcorrective operative diagnosis of a problem, which means that theoretical knowledge of OCD can be achieved only after a series of solution-based strategies have been applied, resulting effectively in addressing the symptoms. In other words, knowing a reality through the strategies that can change it. The fundamental concept of BST is that when a problem or difficulty arises, patients try to solve it, either relying on past experiences by reapplying solutions that have been successful in solving a similar situation in the past, or by attempting new strategies. If these expedients do not work, rather than making use of alternative solutions, the natural tendency is to reiterate them, giving rise to a complex process of retroactions which maintain or exacerbate, instead of modify, the problematic situation. So, the 'attempted solutions' themselves become a problem. Thus, psychological problems are the result of a dysfunctional or pathogenic perceptive-reactive system, ${ }^{23}$ defined as a 'redundant modality through which a given individual perceives and consequently reacts to his own reality in relationship with himself, with others, and with the world' (ref. 24, p. 23).

The strategic psychotherapist is not interested in discovering why a problem exists, but how it is maintained in the present, promoting therapeutic change by applying specific intervention strategies. ${ }^{25}$ Strategic protocols represent rigorous sequences of therapeutic manoeuvres with heuristic and predictive power. Like a game of chess, the therapy becomes a process of strategic problem solving where the experienced players always keep in mind which strategy will lead to a checkmate as he responds to the adversary's moves. The potential reaction to each manoeuvre is predicted and strategical are changes planned on the basis of the observed effects through an ongoing self-corrective process. Since every human interaction, including the therapeutic one, is meant to be unique and unrepeatable, the BST therapist continuously adapts his or her logic and language to those of the patient, promoting flexible, individually tailored interventions. ${ }^{26}$

The metaphorical image that best represents the underlying logic of OCD is made from a story by Paul Watzlawick: 'A man claps his hands every ten seconds. Asked about the reason for this strange behavior, he explains: "in order to scare away the elephants.' When told there are no elephants present, the man responds: 'well, there you go. See?'”. The typical perceptive-reactive system of patients with OCD may be fear or pleasure based. Obsessive ideas emerge as often unreasonable repetitive fixations from which individuals cannot free themselves without performing specific compulsive thinking, formulas or ritual actions. ${ }^{11}{ }^{27}$ However, the attempt to take the matter in hand themselves leads the person to lose control over the situation, and compulsions become inevitable. ${ }^{11}$ It is healthy, for example, to be careful not to get dirty and maintain oneself clean, but it is insane to wash for hours and hours and still doubting that it is not enough. Or, before going to bed, it is certainly a good habit to check that doors, taps or gas valves are closed, but it is definitely absurd to return home or wake up several times during the night for further control. It can also be considered appropriate to take measures in order to pass an exam or to face a stressful situation, but it becomes dysfunctional to structure propitiatory rituals without which it is unthinkable to deal with the circumstance. As Samuel Johnson states (1709-1784): 'the chains of habit are too weak to be felt until they are too strong to be broken', and patients with OCD, notoriously resistant to change, usually ask for help when they lose power over their own actions and thoughts and the problem becomes so much diffused as to affect most aspects of their life. Compulsions, of their own nature, are not illogical, and rationalistic explanations do not lead to any therapeutic success. The use of a non-ordinary logic (opposed to the traditional, rationalistic Aristotelian model) then becomes necessary in order to reorient the symptom towards its selfannulment. First, it is conveyed to patients that what they think and do makes sense; they are given the illusion that the therapist knows a more functional way to manage the situation. In other words, the BST professional needs to follow the logic that underlies the patient's ideas and actions. ${ }^{28}$

Nardone and Portelli ${ }^{11}$ defined five reasons that trigger compulsive thoughts and actions: (1) the turning up of a doubt generating the need for reassuring answers; (2) an excess of ideological rigidity as well as extreme moral respect or religious belief; (3) the excess of rational reasoning processes, until they become completely unreasonable; (4) an extreme health prevention that becomes a phobia and (5) the attempt of reducing anxiety and distress generated by a trauma. For each of these reasons, the purpose may be to prevent or repair something that 'might' happen or 'has' happened, respectively, as well as to propitiate or ensure that things continue to go well.

After having discriminated whether the compulsion is phobic or non-phobic based, in order to achieve the pragmatic knowledge of how to build a successful therapeutic intervention, the Brief Strategic model focuses on the patient's attempted solutions, which in the case of a person suffering from OCD are typically represented by: (1) avoidance of situations that cause anxiety; (2) request for help, reassurance or protection from others in the form of delegation of tasks or in seeking assistance for avoiding contact with fearful stimuli and (3) control of anxiety-laden situations through performing rituals: preventive, propitiatory and reparatory. Other important discriminations to be made are: (1) whether the compulsion is represented by repetitive visible actions or remains at a mental level and (2) if the ritual follows a specific sequence, either numerical or analogical (figure 1). 
Perceptive-reactive system - compulsion

\begin{tabular}{c|cc}
\hline \multicolumn{2}{c}{ Phobic - based on fear } & Non phobic - based on pleasure \\
\hline \multicolumn{3}{c}{ Involvement of others } \\
\hline $\begin{array}{c}\text { No } \\
\text { For shame or because others would not } \\
\text { be able to adequately perform the ritual. }\end{array}$ & $-\quad \begin{array}{c}\text { Seeking assistance } \\
\text { Avoiding to do tasks }\end{array}$ \\
\hline \multicolumn{3}{c}{ Reaction } \\
\hline Actions \\
\hline \multicolumn{3}{c}{ Typology of the ritual } \\
\hline Past-oriented \\
\hline Reparative & Thoughts \\
\hline Propitiatory & Sequence of the ritual \\
\hline Yes & Analogical: \\
\hline $\begin{array}{c}\text { Numerical: } \\
\text { based on quantity }\end{array}$ & based on quality & No \\
\hline
\end{tabular}

Figure 1 Discriminating factors of the strategic intervention.

In order to interrupt the attempted solutions which worsen the situation, the brief strategic therapist, through the use of specific therapeutic communication techniques (strategic dialogue), starts restructuring the perception of the patient's reality by the use of a direct form of communication (ie, 'the more you avoid the fearful situation, the more frightening it becomes' or 'the more you ask for help, the more incapable you become. It invalidates you more and more') aimed at instilling the doubt about the correctness of the person's thoughts and actions.

Depending on the structure of the ritual, an essential and unique aspect of BST is having devised five major techniques specifically designed to dismantle the maintenance mechanism of the symptomatology. ${ }^{28}$ Therapeutic prescriptions or injunctions need to be implemented between sessions, in real life, so as to make the patient autonomously learn how to fight his or her obsession and to change their coping actions. ${ }^{11}$

1. When the ritual holds a sequence, and thus is numerical, the intervention proceeds to give the patient a specific numerical preset counter-ritual, which fits the particular pathological ideas and actions leading to a catastrophic change. This is the case of a person who needs to check something a number of times to ensure that it has been done correctly.

2. Progressive violations of the sequence of the ritual, from small to total violation, in order to break the established rigid control.

3. The technique of postponing the ritual to a specific and prescribed time is aimed at making boring, annoying and unpleasant the individuals' main source of pleasure or gratification, which was driven by impulses. This strategy has been proven to be particularly useful with the vomiting syndrome, a compulsion based on pleasure. Once again, the attempted solution of vomiting for weight control after having binged gradually becomes the problem, and the reason it persists lies in the pleasure provided. Since any repressing intervention would only exacerbate the desire to binge and vomit, by altering the spontaneity of the cycle, the interval technique takes away the enjoyment of the liberating act of vomiting, usually accompanied by the feeling of an almost orgasmic urgency, which progressively becomes more difficult and unpleasant. Thus, a ritual based on pleasure is transformed into an act of self-torture.

4. Ritualising the pathological compulsion in specific space and time sets aside during the day, first numerous then progressively reducing this ritualized ritual to 0 , allows the person to take control of it, gradually demolishing the pathology.

5. Introducing ' $a$ small disorder that maintains order', the objective is to break the rigid control until the unstoppable need for the compulsion completely comes to a stop.

A patient who fears contamination, for example, will continuously wash, clean and sterilise himself, his house and other belongings in order to prevent being infected or contaminated. However, once this state is reached, the problem is to maintain it. In this specific case, the use of the strategic dialogue becomes necessary in order to first reframe the patient's rigid parameters, then to prepare him for complying with the idea that to be immune to dirt, he should not search for total cleanliness but should introduce 'a small disorder'. A little bit of dirt then becomes the only way to protect the person from total cleanliness, responsible for the person's increasing fear. ${ }^{11}$ For example, while persuading a patient with OCD to stop his or her pathological rituals will not lead to any therapeutic success, a prescription based on the same logic underlying the problem will turn the force of the symptom against the disorder itself, breaking its perverse balance. If the patient is told: 'every time you enact one of your rituals, you must repeat it five times, exactly five times, no more, no less. You can avoid doing it at all, but if you do it once, you must do it no more and no less than five times', the injunction to ritually repeat the compulsive action paradoxically brings the person to build a different reality from the one characterised by uncontrollable compulsions. ${ }^{23}$ The logical structure of this apparently simple prescription helps to 'lead the enemy go into the attic and remove the ladder': the individual is not asked to avoid executing the ritual but told, if he or she needs to perform it once, he has to do it five times. In this way, the therapist and then the patient assume control over the situation. When the patient literally follows the prescription, he or she will suspend the ritual after a few days, usually not being able to explain why. Fundamental is the way in which the prescriptions are communicated, that is, by the use of redundantly repeated hypnotic linguistic assonances and the adoption of posthypnotic messages expressed in a more marked tone of voice.

\section{Conceptual and pragmatic comparison between CBT and BST}

Similarly to CBT, BST is based on the modern constructivist epistemology according to which individuals actively 
create their own reality in relationship with themselves, the others and the world; it also makes use of specific treatment protocols focused on dialogue and therapeutic prescriptions.

However, CBT derives from the learning theory, whereas the strategic approach bases its assumptions on the theory of change. In other words, while a CBT therapist guides the patient through a process of awareness and volunteer effort to learn how to fight and handle the disease, the BST professional adopts ad hoc therapeutic stratagems in order to create a corrective emotional experience in the person. By doing so, the patient's resistance to change is bypassed and the way in which they perceive and react to their own reality is transformed.

Differentiating between the two therapeutic approaches is also the type of communication and language adopted during the clinical dialogue as well as used for prescribing therapeutic injunctions. In fact, CBT is traditionally characterised by a logical-rational communication, that is, the indicative language typical of the explanation. In contrast, BST language is injunctive and performative, ${ }^{29}$ aimed at making the person feel differently before acting differently through the use of metaphors, anecdotes and stories (figure 2).

\section{STUDY AIMS AND HYPOTHESES}

Since 1990, the clinical application of the BST to the treatment of OCD has been carried out at the Centro di Terapia Strategica (CTS) at Arezzo, Italy, and has progressively led to the development of ad hoc procedures that have shown to be effective in long-term case reports. ${ }^{30}$ However, despite the encouraging clinical evidence, no quantitative study to date has assessed the effectiveness of BST for OCD.

In order to gather the first empirical evidence, the short-term and medium-term effects of BST on OCD symptoms will be investigated within a one-group observational study, a commonly used clinical and population-based research design that allows the study and the optimisation of healthcare interventions in ecological settings. ${ }^{31}$

Three hypotheses will be tested: (1) OCD symptoms will improve to a statistically and clinically significant extent at the end of the BST intervention; (2) the symptoms improvement will be maintained at 3 month follow-up and (3) since depressive symptoms frequently accompany OCD and appear to affect treatment outcome negatively, higher depression levels at baseline will predict lower improvements in OCD symptomatology.

\section{METHODS}

\section{Study population and recruitment}

Study participants will be recruited among the patients with OCD who are referred to the Hesed House, a state community psychotherapy clinic in Dublin, Ireland, for undertaking the BST intervention. The clinic offers statefunded treatment based on ability to pay and generally provides care to lower socioeconomic groups. Patients are referred from two general medical hospitals, the Naas General Hospital and the St James Hospital, and from a Community-based primary care Psychiatric Service, the Carlow, West Wicklow, Mental Health team, all located in Dublin, Ireland, where they undergo a diagnostic assessment and receive primary care by resident psychiatrists. On arrival at the Hesed House, patients will be consecutively screened according to the selection criteria and the eligible ones will be invited to participate in the study by a researcher who will provide them with detailed information about the study aims and procedure. Those who will agree to participate and will sign the informed consent form will be included in the study.

\section{Inclusion and exclusion criteria}

Patients will be considered eligible for the study when they meet the following inclusion criteria: (1) being 18 or over and (2) being assigned a Diagnostic and Statistical Manual of Mental Disorders, Fourth Edition (DSM-IV) or DSM-V diagnosis of OCD by the referring psychiatrist. Exclusion criteria will be: (1) presenting established cognitive or communication problems which makes it challenging to understand the questionnaires and take part in the therapeutic encounters; (2) having vision impairment which makes difficult it to fill in the questionnaires and (3) suffering from other severe psychiatric disorders.

\section{Outcome measures}

The clinical severity of $O C D$ symptoms will be measured by the Yale-Brown Obsessive Compulsive Scale (Y-BOCS) ${ }^{32-34}$ a self-report, 10-item questionnaire extensively used in research and clinical practice to monitor improvement during treatment. The Y-BOCS consists of two parts: the Symptom Checklist for evaluating the presence of current and past symptoms, and the 10-item Severity Scale (rated 0-4 per item) that assesses obsessions and compulsions separately in five dimensions (time spent, interference, distress, resistance and control). Separate Obsession (items 1-5) and Compulsion (items 6-10) subscale scores (range 0-20) are summed to yield a total Y-BOCS score (range 0-40)..$^{35}$ Depressive symptoms will be assessed by the Beck Depression Inventory-II (BDI-II), ${ }^{36} 37$ a 21-question, multiple-choice, self-report inventory widely used for measuring the severity of depression.

\section{Sample size}

Twenty-eight patients at least will be included in the study. This minimum sample size was calculated considering the possible violation of the normality assumption and will give the one-tailed, non-parametric Wilcoxon signed-rank test sufficient statistical power $(80 \%)$ to detect a medium-sized within-subject effect $(d=0.5)$. If parametric assumptions will be satisfied, an equal number of participants will give the one-tailed, pairedsample Student $t$ test a bit higher statistical power $(82 \%)$ 


\section{SIMILARITIES}

- They are based on the modern constructivist epistemology that believes the subject being an active builder of his or her own reality and not a mere victim of it.

- They make use of specific protocols of intervention focused on dialogue and therapeutic prescriptions.

- The patient works both during the therapeutic encounters, together with the professional, and alone, between sessions.

- Proven effectiveness in treating OCD in short time.

- Require a good client-therapist relationship.

- Emphasize collaboration and active participation.

- Are focused on the present.

- Sessions are structured.

\begin{tabular}{|c|c|}
\hline \multicolumn{2}{|c|}{ DIFFERENCES } \\
\hline BST & CBT \\
\hline Derives from the theory of change. & Derives from the theory of learning. \\
\hline Represents a solution-focused approach. & Represents a problem-focused approach. \\
\hline $\begin{array}{l}\text { Therapist uses stratagems which create real } \\
\text { corrective emotional experiences in the way the } \\
\text { persons perceive and react to their own reality, } \\
\text { leading them to acquire the skills required to } \\
\text { autonomously cope with the situation. } \\
\text { Change } \rightarrow \text { Consciousness }\end{array}$ & $\begin{array}{l}\text { Therapist guides the patient through a process } \\
\text { based on the awareness and voluntary effort to } \\
\text { learn how to fight or manage the disorder. } \\
\text { Consciousness } \rightarrow \text { Change }\end{array}$ \\
\hline $\begin{array}{l}\text { Resistances is bypassed by using stratagems that } \\
\text { create a change beyond the voluntary effort of } \\
\text { the } \\
\text { patient. }\end{array}$ & $\begin{array}{l}\text { Therapist goes through the willingness of the } \\
\text { subject, often stumbles by the resistance to } \\
\text { change which can be strong. }\end{array}$ \\
\hline $\begin{array}{l}\text { Methodology of research: working for effects of } \\
\text { discovery and subsequent acquisitions. }\end{array}$ & $\begin{array}{l}\text { Methodology of research: working for } \\
\text { progressive acquisition of knowledge. }\end{array}$ \\
\hline $\begin{array}{l}\text { Change occurs quickly by unlocking the } \\
\text { symptomatology in a way that may appear } \\
\text { almost magical to the person. }\end{array}$ & $\begin{array}{l}\text { Change happens gradually by helping the } \\
\text { patients acquiring the abilities required for } \\
\text { controlling their thoughts and actions. }\end{array}$ \\
\hline $\begin{array}{l}\text { Relapses are seen as opportunity to revise } \\
\text { ongoing strategies. }\end{array}$ & Emphasizes relapse prevention. \\
\hline $\begin{array}{l}\text { Communication is performative and injunctive, } \\
\text { Hypnotic and evocative language is used to } \\
\text { makes the patient feel even before to } \\
\text { understand. Both logic and analog languages are } \\
\text { employed, together with suggestive metaphors } \\
\text { as well as verbal and non-verbal hypnotic } \\
\text { communication. }\end{array}$ & $\begin{array}{l}\text { Communication is logical-rational, and make } \\
\text { use of the indicative language typical of the } \\
\text { explanation. }\end{array}$ \\
\hline $\begin{array}{l}\text { The therapist assumes a position which must be } \\
\text { complementary to the problem of the patient to } \\
\text { avoid in-session's confrontations, to put the }\end{array}$ & $\begin{array}{l}\text { The therapist assumes a direct, one-up position } \\
\text { toward the patient. }\end{array}$ \\
\hline $\begin{array}{l}\text { patient at ease so s/he can open up, to bypass } \\
\text { resistance, to establish therapeutic alliance, to } \\
\text { motivate the person to do something different, } \\
\text { and to reinforce even small successes. }\end{array}$ & \\
\hline The therapy is adapted to the patient. & The patient must follow the therapeutic rules \\
\hline $\begin{array}{l}\text { The therapeutic encounters can last up to } 3 \\
\text { hours. }\end{array}$ & $\begin{array}{l}\text { The therapeutic encounters do not last more } \\
\text { than } 45-60 \text { minutes. }\end{array}$ \\
\hline $\begin{array}{l}\text { The patient is responsible for the success of the } \\
\text { therapy. }\end{array}$ & $\begin{array}{l}\text { The responsibility for the therapeutic success is } \\
\text { of the therapist. }\end{array}$ \\
\hline
\end{tabular}

Figure 2 Comparison between CBT and BST. BST, brief strategic therapy; CBT, cognitive-behavioural therapy; OCD, obsessive-compulsive disorder. 
to detect the same medium-sized within-subject effect. $\mathrm{G}^{*}$ Power (V.3.1.3) was used for calculations.

\section{Study flow}

Before starting treatment, participants will be administered the study questionnaires in a quiet room under the supervision of an independent psychometrician. Treatment will take place every 2 weeks in a face-to-face setting and will consist of 1045 min BST sessions. At the end of the last one, participants will be readministered the study questionnaires and those showing a clinically significant reduction of the Y-BOCS total score will be contacted after 3 months for the follow-up assessment, which will consist in sending the study questionnaires to participants by mail and in asking them to send them back after having answered all items. The clinical significance of the Y-BOCS total score reduction will be assessed by means of the Jacobson and Truax method. ${ }^{38}{ }^{39}$ Conversely, participants not showing a significant reduction of the Y-BOCS total score at the end of the last planned session will continue to be enrolled in the therapy and will be excluded from the follow-up study phase.

\section{Psychotherapists and treatment fidelity}

Therapists who deliver the BST treatment at the Hesed House are all trained in BST and specifically qualified for the treatment of OCD. The therapy process will be monitored and live video cases will be supervised by a BST senior clinician.

\section{Data analysis}

The one-tailed, paired-sample Student t test will be used to test the statistical significance of change in Y-BOCS scores between baseline and treatment termination. However, if data will strongly violate the parametric assumptions, then the non-parametric Wilcoxon test will be used. The second hypothesis (the maintenance of OCD symptoms improvement at the 3 month follow-up) will be tested by the equivalence testing method, ${ }^{40}$ which will be also used to assess the clinical significance of the BST effect. This will be accomplished by determining if the study group will be equivalent to the normative sample after the BST intervention and at follow-up. Finally, the hypothesis that higher depression levels at baseline are predictive of lower reductions in OCD symptomatology at treatment termination will be tested by means of simple regression. All data analyses will be performed using SPSS V.22 (SPSS, Inc, Chicago, Illinois, USA).

\section{Patients withdrawal and missing data analysis}

Study participants may stop the treatment for any reason at any time. In addition, they may stop participating in the study and withdraw all consents. Reasons for withdrawal will be investigated and reported only for withdrawing participants giving the consent to communicate them. Missing data at treatment termination and at follow-up will be first inspected for determining the missingness pattern. Only data missing at random will be then imputed by multiple imputation.

\section{DISSEMINATION}

All participants provide written informed consent before answering the baseline questionnaires and may withdraw at any point. Findings will be published in peer-reviewed journals and presented at conferences. Authorship will follow the criteria recommended by the International Committee of Medical Journal Editors.

\section{CONCLUSIONS}

The research-intervention programme carried out for $>15$ years by the CTS of Arezzo, Italy, has led to the development of specific treatment protocols for a series of psychopathological disorders, and the clinical evidence that has been gathered over time supports the hypothesis that BST is highly efficacious in treating OCD. Indeed, clinical evidence shows that even the most obstinate obsessions and compulsions are usually won over by redefining the situation and by setting up a series of concrete corrective emotional experiences that free the patient from a rigid self-feeding perceptive-reactive system. Focusing on the individual attempted solutions, then understanding what maintains and worsens the problem, the strategic approach is essentially aimed at creating a corrective emotional experience, transforming the way in which the person perceives and reacts to his own reality. Through the use of ad hoc therapeutic stratagems and in-session injunctive and performative language, BST bypasses the individual usual rational mechanisms, leading to the self-destruction of the logic that imprisons the mind, then quickly interrupting the vicious cycle that maintains the problem.

Located in the tradition of Lewin, the action research method typically used for investigating the effectiveness of BST in treating diverse forms of psychopathologies refers to the long-term stability of the therapeutic outcomes, as assessed by both the therapist and the patient through a change-related rating scale, with respect to the therapeutic goal. Empirical data also refer to how many therapists apply a certain protocol in their daily practice on real patients. BST effectiveness is related to the complete extinction of the symptomatology, which is tested in follow-up encounters usually scheduled after 3, 6, 9 and 12 months after the treatment termination (for OCD, once phobic symptoms and compulsive beliefs are eliminated). Since, by its own nature, BST is aimed at solving complex problems in a short time, OCD symptoms release may be obtained even within the first encounters. This aspect should not be underestimated. In fact, even though the efficacy of CBT in treating OCD symptoms has been proven in several investigations, ${ }^{41-43}$ it is different to be free from a debilitating disease in $2 / 3$ months instead of $2 / 3$ years. The efficiency of a treatment underlines the real therapeutic efficacy. 
The BST research-intervention approach has been shown to be a valid method for acquiring operative knowledge on OCD. However, no empirical study has been conducted as yet. For this reason, an observational study aimed at empirically assessing the effect of the OCD-specific BST has been planned. Since it represents the first investigation that will statistically test the effects of the BST protocol for OCD, future controlled trials are required in order to better evaluate both the efficacy and the effectiveness of either BST alone or in combination with other treatment strategies in treating obsessive and compulsive symptoms.

\section{Author affiliations}

${ }^{1}$ Psychology Research Laboratory, Ospedale San Giuseppe, IRCCS, Istituto Auxologico Italiano, Oggebbio (VCO), Italy

${ }^{2}$ Department of Psychology, Catholic University of Milan, Italy

${ }^{3}$ Faculty of Psychology, eCampus University, Novedrate, Como, Italy

${ }^{4}$ Bateson Clinic, Dublin, Ireland

${ }^{5}$ Dublin City University, Ireland

${ }^{6}$ The OCD Clinic Dublin, Ireland

${ }^{7}$ Strategic Therapy Center, Arezzo, Italy

${ }^{8}$ Hesed House, Dublin, Ireland

${ }^{9}$ Department of Education and Psychology, University of Florence, Italy

Acknowledgements The authors would like to express their sincere thanks to Centro di Terapia Strategica (CTS)—Brief Strategic Center, Arezzo, Italy for provision of expertise. Without their superior knowledge and experience, the Project would lack in quality of outcomes, and thus their support has been essential.

Contributors GP, GMM, PG, DB and GC conceived of the study. GMM and AG provided statistical expertise in study design and will conduct the statistical analysis. All the authors contributed to refinement of the study protocol and approved the final manuscript.

Competing interests None declared.

Patient consent Obtained.

Ethics approval The study was approved by the Hesed House Ethics Board in Dublin.

Provenance and peer review Not commissioned; externally peer reviewed.

Open Access This is an Open Access article distributed in accordance with the Creative Commons Attribution Non Commercial (CC BY-NC 4.0) license, which permits others to distribute, remix, adapt, build upon this work noncommercially, and license their derivative works on different terms, provided the original work is properly cited and the use is non-commercial. See: http:// creativecommons.org/licenses/by-nc/4.0/

\section{REFERENCES}

1. Keeley M, Storch EA. The nature, assessment, and treatment of pediatric obsessive-compulsive disorder. Behav Psychol 2008;16:535-51.

2. American Psychiatric Association. Diagnostic and Statistical Manual of Mental Disorders. 4th edn. Washington: American Psychiatric Press, 1994.

3. Karno M, Golding JM, Sorenson SB, et al. The epidemiology of obsessive-compulsive disorder in five US communities. Arch Gen Psychiatry 1988;45:1094-9.

4. Abramowitz JS, Taylor S, McKay D. Obsessive-compulsive disorder. Lancet 2009;374:491-9.

5. Foa EB. Cognitive behavioral therapy of obsessive-compulsive disorder. Dialogues Clin Neurosci 2010;12:199-207.

6. Geffken GR, Storch EA, Gelfand KM, et al. Cognitive-behavioral therapy for obsessive-compulsive disorder: review of treatment techniques. J Psychosoc Nurs Ment Health Serv 2004;42: 44-51
7. Murray CJ, Lopez AD. Global mortality, disability, and the contribution of risk factors: global burden of disease study. Lancet 1997;349:1436-42.

8. Torres AR, Fontenelle LF, Ferrão YA, et al. Clinical features of obsessive-compulsive disorder with hoarding symptoms: a multicenter study. J Psychiatr Res 2012;46:724-32.

9. Doron G, Moulding R, Kyrios M, et al. Sensitivity of self-beliefs in obsessive compulsive disorder. Depress Anxiety 2008;25: 874-84.

10. Keeley ML, Storch EA, Merlo LJ, et al. Clinical predictors of response to cognitive-behavioral therapy for obsessive-compulsive disorder. Clin Psychol Rev 2008;28:118-30.

11. Nardone G, Portelli C. Ossessioni compulsioni manie. Capirle e sconfiggerele in tempi brevi. Milano: Ponte delle Grazie, 2013.

12. Storch EA, Abramowitz J, Goodman WK. Where does obsessive-compulsive disorder belong in DSM-V? Depress Anxiety 2008;25:336-47.

13. Abramowitz JS. Effectiveness of psychological and pharmacological treatments for obsessive-compulsive disorder: a quantitative review. J Consult Clin Psychol 1997;65:44-52

14. [No authors listed]. Treatment of obsessive-compulsive disorder. Expert Consensus Panel for Obsessive-Compulsive Disorder. J Clin Psychiatry 1997;58(Suppl 4):2-72.

15. van Oppen $P$, de Haan E, van Balkom AJ, et al. Cognitive therapy and exposure in vivo in the treatment of obsessive compulsive disorder. Behav Res Ther 1995;33:379-90.

16. Abramowitz JS. The psychological treatment of obsessivecompulsive disorder. Can J Psychiatry 2006;51:407-16.

17. Doron $\mathrm{G}$, Kyrios M. Obsessive compulsive disorder: a review of possible specific internal representations within a broader cognitive theory. Clin Psychol Rev 2005;25:415-32.

18. Meyer V. Modification of expectations in cases with obsessional rituals. Behav Res Ther 1966;4:273-80.

19. Lewin $A B, D e$ Nadai $A S$, Park J, et al. Refining clinical judgment of treatment outcome in obsessive-compulsive disorder. Psychiatry Res 2011;185:394-401.

20. Geller DA, Biederman J, Stewart SE, et al. Impact of comorbidity on treatment response to paroxetine in pediatric obsessive-compulsive disorder: is the use of exclusion criteria empirically supported in randomized clinical trials? J Child Adolesc Psychopharmacol 2003;13(Suppl 1):S19-29.

21. Storch EA, Björgvinsson T, Riemann B, et al. Factors associated with poor response in cognitive-behavioral therapy for pediatric obsessive-compulsive disorder. Bull Menninger Clin 2010;74:167-85.

22. Essays UK. Treatment of Obsessive Compulsive Disorder Psychology Essay. 2013. http://www.ukessays.com/essays/ psychology/treatment-of-obsessive-compulsive-disorderpsychology-essay.php?cref $=1$

23. Nardone G, Portelli C. Knowing through changing: the evolution of brief strategic therapy. Glasgow: Crown House Publishing, 2005.

24. Nardone G, Balbi E. Solcare il mare all'insaputa del cielo. Lezioni sul cambiamento terapeutico e le logiche non ordinarie. Milano: Ponte alle Grazie, 2008.

25. Nardone G, Verbitz T, Milanese R. The prisons of food: strategic solution-oriented research and treatment of eating disorders. London: Karnac Publishing, 1999.

26. Nardone G, Watzlawick P. Brief strategic therapy: philosophy technique and research. Lanham, MD: Jason Aronson, 2005.

27. Portelli C. Advanced brief strategic therapy for obsessive-compulsive disorders. Brief Strateg Syst Ther Eur Rev 2004;1:88-97.

28. Portelli C. Brief strategic interventions for obsessive compulsive disorders: acquiring the maximum with the minimum in the first session. Brief Strat Syst Ther Eur Rev 2005;2:56-70.

29. Austin JL. How to do things with words. Cambridge: Harvard University Press, 1962.

30. Nardone G, Watzlawick P. L'arte del Cambiamento: la soluzione dei problemi psicologici e interpersonali in tempi brevi. Milano: Ponte alle Grazie, 1990.

31. Embi PJ, Kaufman SE, Payne PR. Biomedical informatics and outcomes research: enabling knowledge-driven health care. Circulation 2009;120:2393-9.

32. Woody SR, Steketee G, Chambless DL. Reliability and validity of the Yale-Brown Obsessive-Compulsive Scale. Behav Res Ther 1995;33:597-605

33. Kim SW, Dysken MW, Kuskowski M. The Yale-Brown ObsessiveCompulsive Scale: a reliability and validity study. Psychiatry Res 1990;34:99-106.

34. Tek C, Ulug B, Rezaki BG, et al. Yale-Brown Obsessive Compulsive Scale and US National Institute of Mental Health Global Obsessive Compulsive Scale in Turkish: reliability and validity. Acta Psychiatr Scand 1995;91:410-13. 
35. Seol SH, Kwon JS, Shin MS. Korean self-report version of the Yale-Brown Obsessive-Compulsive Scale: factor structure, reliability, and validity. Psychiatry Investig 2013;10:17-25.

36. Wang YP, Gorenstein C. Psychometric properties of the Beck Depression Inventory-II: a comprehensive review. Rev Bras Psiquiatr 2013;35:416-31.

37. Beck AT, Ward $\mathrm{CH}$, Mendelson $\mathrm{M}$, et al. An inventory for measuring depression. Arch Gen Psychiatry 1961;4:561-71.

38. Jacobson NS, Truax P. Clinical significance: a statistical approach to denning meaningful change in psychotherapy research. $J$ Consult Clin Psychol 1991;59:12-19.

39. Jacobson NS, Roberts LJ, Berns SB, et al. Methods for defining and determining the clinical significance of treatment effects: description, application, and alternatives. J Consult Clin Psychol 1999;67:300-7.
40. Manzoni GM, Cribbie RA, Villa V, et al. Psychological well-being in obese inpatients with ischemic heart disease at entry and at discharge from a four-week cardiac rehabilitation program. Front Psychol 2010;1:38.

41. Murray K, Jassi A, Mataix-Cols D, et al. Outcomes of cognitive behaviour therapy for obsessive-compulsive disorder in young people with and without autism spectrum disorders: a case controlled study. Psychiatry Res 2015;228:8-13.

42. Aslam M, Irfan M, Naeem F. Brief culturally adapted cognitive behaviour therapy for obsessive compulsive disorder: a pilot study. Pak J Med Sci 2015;31:874-9.

43. Oldfield VB, Salkovskis PM, Taylor T. Time-intensive cognitive behaviour therapy for obsessive-compulsive disorder: a case series and matched comparison group. Br J Clin Psychol 2011;50:7-18. 